\title{
Similarities and differences in nurse-reported care rationing between critical care, surgical and medical specialities
}

\author{
Megan H Higgs \\ RN, MN, PhD Candidate \\ Ritin Fernandez \\ RN, MN (Critical Care), PhD \\ Suzanne Polis \\ RN, MPH (Research), PhD Candidate \\ University of Technology Sydney, Faculty of Health \\ Sydney Children's Hospital Network-Sydney Children's Hospital \\ Vicki Manning \\ RN, MPH, BAdmin (Nursing) \\ St George Hospital, Executive Unit, Kogarah, New South Wales, Australia
}

St George Hospital, Centre for Research in Nursing and Health, Kogarah, New South Wales, Australia

St George Hospital, Centre for Research in Nursing and Health, Kogarah, New South Wales, Australia

School of Nursing, Faculty of Science, Medicine and Health, University of Wollongong, Australia

Disclosures: No funding was received for this project and there are no conflicts of interest to disclose.

Corresponding author:

M. Higgs

Address: Centre for Research in Nursing and Health

Level 1 Research and Education Building

St George Hospital

Gray Street

Kogarah, 2217

Sydney, New South Wales

Australia

Email: Megan.Higgs@health.nsw.gov.au

Phone: 91131200

Fax: 91134113 


\title{
Similarities and differences in nurse-reported care rationing between critical care, surgical and medical specialities
}

\begin{abstract}
Objective: To determine the similarities and differences in elements of nursing care that are commonly rationed and the reasons why such rationing occurs in the critical care, medical and surgical specialities within an acute hospital environment.
\end{abstract}

Methods: Registered nurses who provide bedside nursing care within the medical, surgical and critical specialities at a single centre were invited to anonymously complete the self-administered MISSCARE questionnaire.

Results: Interventions related to basic care was the most frequently rationed care group in the critical care/emergency specialty while planning was the most frequently rationed care group among both the medical and surgical specialties. Assessment was the least frequently rationed care group amongst all three specialties. Length of time practising as a registered nurse was an independent predictor of care rationing in the critical care/emergency specialty and age greater than 50 was an independent predictor in the medical specialty.

Conclusion: There are numerous similarities and differences in care rationing between critical care, surgical and medical nurses. Individualized strategies based on the type of speciality should be developed to reduce the incidence of nursing care rationing. 


\section{Introduction}

Increasing demand for health services coupled with limited availability of health care resources has led to the rationing of health care globally. ${ }^{1-3}$ Rationing of care has been reported extensively in the health literature and is not new to nursing. ${ }^{3-5}$ Methods of rationing nursing care involve providing care that is vital and withholding care that is not essential without potentially compromising patient safety. ${ }^{6}$ The term "missed care" is widely used in the literature to describe care that is not provided by nurses during their shift. ${ }^{7-12}$

Nurses play a fundamental role in the provision of patient care within the hospital environment. They attend to a large proportion of patient care, performing interventions that are prescribed by other members of the multidisciplinary team as well as nurse initiated interventions. ${ }^{13}$ The care that is provided by nursing staff within the hospital environment is multidimensional, including not only the multiple direct patient care activities that are performed at the bedside but indirect patient care activities that include planning, co-ordination, education documentation and emotional support, as well as non-nursing specific tasks such as locating equipment and supplies, housekeeping and cleaning. This workload along with increased patient acuity ${ }^{14}$, lack of resources ${ }^{15}$, and staff shortages ${ }^{16}$ have been the key reasons for nurses' decisions to ration the care provided each shift.

There were a total of 9.7 million hospital discharges in Australia during the 2013-14 financial year, the majority of which, $92 \%$, were categorized as episodes of acute care. ${ }^{17} \mathrm{~A}$ comparison of data from $2009-10$ demonstrates that our hospitals have seen an approximate one million increase in the number of acute care discharges over the past 5 years, while patient length of stays have steadily decreased ${ }^{17}$ indicating that nurses within the acute care hospital environment are required to deliver complex care within a shorter period of time. In addition, increasing hospital admissions due to the aging population, disease chronicity and the presence of co-morbidities has made the shift work busy and unpredictable, resulting in a necessity for nurses to ration nursing care.

A review of the current literature demonstrates that care that is generally rationed is related to basic care. Frequently rationed elements of care include answering call bells, showering patients, turning patients, monitoring vital signs and changing soiled bed linen. ${ }^{18}$ A large study undertaken on 33659 nurse's across 488 hospitals in 12 European countries indicated that the most frequent nursing care activities that are rationed 
included comfort/talk with patients, developing or updating nursing care plans/care pathways and educating patients and families. ${ }^{5}$ In addition, a systematic review of 17 quantitative studies identified that communication with patients and families, patient ambulation, and mouth care were common elements of care that are rationed by nurses. ${ }^{3}$ Data obtained from 729 patients in a multi-site study similarly implicated care activities within the basic care category as being the most frequently rationed, with mouth care, ambulation, sitting patients out of bed and bathing being identified as frequently missed elements of care. ${ }^{19}$

There is extant literature on the association between rationing nursing care and adverse patient outcomes. These adverse outcomes include patient falls, ${ }^{16}$ pressure ulcers, ${ }^{20}$ medication errors, ${ }^{20}$ nosocomial infections ${ }^{20}$, patient dissatisfaction with care ${ }^{4,21,22}$ and increased readmissions to hospital. ${ }^{23}$ A study that evaluated the patient perspective of missed nursing care demonstrated this correlation between high levels of care rationing and the development of adverse outcomes, with patients who reported experiencing an adverse outcome reporting higher levels of missed care than those patients who did not report an adverse outcome. ${ }^{19}$

Nurse to patient ratios and staff mix are environmental factors that have been identified as having a direct impact on care rationing decisions. A significant correlation was found between nurse to patient ratio in a large scale, multi-site study in England with the findings showing that nurses looking after six or fewer patients missed significantly less elements of care $(\mathrm{p}<0.01)$ than nurses looking after 11 or more patients, with an exponential trend in the frequency of missed care as the patient to nurse ratio increased. ${ }^{24}$ In another large scale, multi-site study carried out in Europe similar findings were ascertained with nurse reported missed care being significantly less in hospitals with lower patient to nurse ratios $(\mathrm{p}<0.0001)$ and when nurses were carrying out non-nursing tasks less frequently $(\mathrm{p}<0.0001)^{5}$

To ration the care they provide, nurses must draw upon their clinical judgement to appropriately prioritize patient care needs without compromising the quality of care provided or patient safety. ${ }^{25}$ These decision making processes can pose nurses with an ethical dilemma ${ }^{26}$ and have been reported to cause nurses significant distress leading to burn out and an exodus from the nursing profession. ${ }^{27,28}$ An understanding of the care rationing patterns of nursing staff and the reasons for the need for such rationing can assist in the development and 
implementation of strategies to decrease the necessity of care rationing, therefore reducing the incidence of adverse patient outcomes and nursing burnout.

This study was a sub-study of a large nursing workforce survey and aimed to determine the similarities and differences in elements of care that are commonly rationed and the reasons why such rationing occurs in the critical care, medical and surgical specialities within an acute hospital environment.

\section{Method}

A detailed description of the study methods has been reported elsewhere. ${ }^{29}$ In brief, data from all registered nurses who provide bedside nursing care at a 650 bedded tertiary referral hospital in Sydney were included in the study. The nursing workforce at the hospital comprises of $97 \%$ of registered nurses and $3 \%$ enrolled nurses. Participants were required to complete a self-administered, anonymous, cross sectional survey.

The questionnaire utilised to measure rationing of nursing care in this study was an adapted version of the previously validated and reliable MISSCARE scale. ${ }^{30}$ The original MISSCARE scale comprized of twenty two common nursing care elements relating to Assessment, Intervention - Individual needs, Intervention - Basic Care and Planning and measures the perceived frequency of which elements of care within these areas are missed. In this study following extensive discussions with clinical and research experts, three items that were important nursing care elements within the local setting of the study were added to the original 22 items. The three additional items included were; skin/wound care, falls risk assessment completed within twenty-fours of admission and falls prevention strategies implemented. For each of the twenty five items participants were asked to rate the items on a five point Likert scale $(1=$ always missed, $2=$ frequently missed, $3=$ occasionally missed, $4=$ rarely missed and $5=$ never missed).

Participants were instructed to complete the questionnaire as a reflection of their wards practice environment, i.e. the frequency of care omissions by nursing staff (including themselves) on their ward and reasons such omissions occur on their ward. In addition, participants completed a seven item demographic questionnaire. 
Participation in the study was voluntary and consent to participate was implied by the return of a completed survey. Participants were informed about the survey through in-service education and letters of invitation disseminated by the nursing unit manager of each department. Surveys along with a dedicated and secure collection box were then distributed to each department by members of the research team. Participants were allotted time during their shift to complete the survey. Completed surveys were routinely collected by research personnel and the raw data entered into an excel database. Ethics approval for this study was obtained from the South Eastern Sydney Local Health District Human Ethics Research Committee.

\section{Data Analysis}

All data analysis was carried out using IBM Statistical Package for the Social Sciences (SPSS) version 21, Armonk, New York. Prior to data analysis all twenty five items within Part A of the questionnaire were recoded to form a dichotomous scale. As such the responses "never missed" and "rarely missed" were recoded to demonstrate a negative response (care item not missed) and the responses "occasionally missed", "frequently missed" and "always missed" were recoded to demonstrate a positive response (care item missed). Each item was then examined with frequencies and percentages. An overall mean of the percentage of missed care, inclusive of all items, was determined for each sub-group. Results were stratified by speciality, i.e. critical care/emergency, medical and surgical.

The total missed care score was calculated as the sum of the 25 individual items, using the five point scale. Univariate descriptive statistics by speciality (critical care/emergency, medical and surgical) were undertaken to determine the participant characteristics. Distributions were examined for violations of normality for parametric procedures. Participants' age was examined using four categories: 20-29 years, 30-39 years, 40-49 years and 50 and greater. Participants' highest level of education was examined using four categories: Less than a Bachelor's degree, Bachelor's degree, Post Graduate Certificate or Diploma, Master's degree or PhD. Differences between the four groups were investigated using one-way analysis of variance (ANOVA) followed by Bonferroni correction to identify the groups that were significantly different from one another. Missing data for ANOVA was handled with listwise deletion. All variables that were statistically significant in the univariate analysis were included in a standard multiple linear regression analysis to determine the factors that were independently associated with overall missed care. Participants' age and highest level of education were coded as dummy 
variables. 'Length of time working as a registered nurse' and 'length of time working in the current ward' was entered as a continuous variable. Statistical significance was set at $\mathrm{p}<0.05$. Regression analysis was carried out by speciality, i.e. critical care/emergency, medical and surgical.

\section{Results}

A total of 249 registered nurses who provide direct patient care completed the survey of whom 87 worked within the critical care/emergency speciality, 89 within medical and 73 within surgical. Within each specialty the majority of participants were female $(>80 \%)$ and the majority of participants were 30 years of age and over (approx. 55\%). The highest level of education held by the majority of participants in each specialty was a Bachelor's degree. A higher percentage of participants within the critical care speciality (39.3\%) had completed post graduate studies compared to the medical (15.9\%) and surgical $(9.7 \%)$ specialties. Employment status was predominantly full time $(>70 \%)$ and the majority of respondents worked a rotating roster $(>80 \%)$. The mean length of time reported as working as a registered nurse varied amongst specialties ranging from 7.9 years in the medical specialty to 11.5 years in critical care. Participants had been employed on their study wards for an average of four to seven years.

\section{Rationing of elements of care}

\section{$\underline{\text { Assessment }}$}

Overall, assessment was more frequently rationed in the critical care/emergency specialty $(26.7 \% \pm 15.5)$ than the medical $(23.1 \% \pm 12.2)$ and surgical specialties $(19.7 \% \pm 14.7)$ and it was the least frequently rationed care group amongst all three specialties (Table 2). The element of care 'falls risk assessment conducted within 24 hours of admission' was the most frequently rationed element or care within both the critical care/emergency (62.8\%) and surgical specialties (50.7\%). The element of care 'patient assessments performed each shift' was the most frequently rationed element within the medical specialty $(44.2 \%)$ and the second most rationed element within the surgical specialty (32.4\%). 'Focused reassessment according to patient condition' was reported to be more frequently rationed in the medical $(30.2 \%)$ and surgical $(22.9 \%)$ specialties than the critical care/emergency specialty (15.5\%). The elements of care 'hand washing' and 'monitoring input/output' were reported to be rationed more frequently in the critical care/emergency specialty $(30.6 \% ; 34.1 \%)$ than the medical $(8.1 \% ; 27.9 \%)$ and surgical specialties $(4.4 \% ; 17.6 \%)$ respectively. Reports of rationing for the care 
element 'IV/central line site care and assessments according to hospital policy' ranged from $20.6 \%$ (surgical) to $31.4 \%$ (medical).

$\underline{\text { Intervention - individual needs }}$

The elements of care within the intervention related to individual needs care group, 'falls prevention strategies implemented', 'assist with toileting needs within 5 minutes of request' and 'emotional support to patient and/or family' had the highest frequency of rationing within the critical care/emergency and surgical specialties.

Similarly, the medical specialty had high reports of care rationing for the element of care 'assist with toileting needs within 5 minutes of request' $(61.6 \%)$. In contrast to critical care/emergency and surgical specialties the medical specialty reported the highest frequencies of rationing in the elements 'PRN medication request acted on within 15 minutes' and 'assess effectiveness of medications'

\section{$\underline{\text { Intervention - basic care }}$}

Interventions related to basic care was the most frequently rationed care group in the critical care/emergency specialty (50.1\%) (Table 2). Among this care group the elements of care 'ambulation 3 times per day as required', 'turning patient every two hours', 'mouth care' and 'feeding patient when the food is still warm' were the most frequently rationed elements reported in the critical care/emergency specialty. In contrast the elements of care 'skin/wound care', 'patient bathing/skin care' and 'setting up meals for patient who feeds themselves' were the most frequently rationed elements reported by both the medical and surgical specialties.

\section{$\underline{\text { Planning }}$}

Planning was the most frequently rationed care group among both the medical and surgical specialties, $43.6 \% \pm$ 16.4 and $44.8 \% \pm 18.8$ respectively (Table 2 ). 'Patient teaching about illness, tests and diagnostic studies' and 'patient discharge planning and teaching' were the most frequently rationed elements of care related to planning in both medical and surgical specialties. In contrast 'attend interdisciplinary care conferences whenever held' was the most frequently rationed planning care element reported in the critical care/emergency specialty.

\section{Predictors of missed care}

\section{$\underline{\text { Critical Care }}$}


Within the critical care and emergency specialty greater amount of care rationing was identified in participants aged $>50$ years and those with their highest level of education being a graduate certificate or diploma. However the differences were not significant. The longer participants had been practising as a registered nurse, and the longer the length of time spent on the current ward, the greater the amount of care rationing. However, only length of time practising as a registered nurse had a significant correlation. (Table 3 )

For the critical speciality the only significant variable; "length of time working as a registered nurse', was included in the regression model. The linear regression model to predict missed care was significant and accounted for $6.6 \%$ of the variance $\left(\mathrm{R}^{2}=0.078, \mathrm{R}^{2} \mathrm{Adj}=0.066, \mathrm{~F}(1,82)=6.9, \mathrm{P}=0.010\right.$ ( table 4$)$. The results indicate that missed care was greater among those who worked a longer time as a registered nurse.

\section{Medical}

Participant age $>50$ in the medical specialty demonstrated a significantly greater amount of care rationing than the three other age categories, similarly participants whose highest level of education was less than a bachelor's degree demonstrated significantly higher amounts of care rationing than those participants who held a bachelor's degree or post graduate qualification. The length of time participants had been practising as a registered nurse, and working on the current ward, was significantly related to greater the amount of care rationing. (Table 3)

For the Medical speciality the following predictor variables that were significant were included in the model: age 50 years and greater, less than a Bachelor's degree, 'length of time working as a registered nurse' and 'length of time working in the current ward'. The multiple regression model to predict missed care was significant and accounted for $6.6 \%$ of the variance $\left(R^{2}=0.289, R^{2} A d j=0.212, F(8,73)=3.71, P=0.01\right.$. The unique contribution of each variable $\left(\mathrm{sr}^{2}\right)$ in the final model is presented in Table 4. Only one variable length of time working as a registered nurse was significant and was independently related to missed care.

\section{$\underline{\text { Surgical }}$}

Within the surgical specialty participant age 20-29 demonstrated a greater amount of care rationing than the three other age categories and similarly to the critical care/emergency specialty those with their highest level of education being a graduate certificate or diploma in the surgical specialty demonstrated a greater amount of care 
rationing than all other levels of education. The differences between groups for both variables were not significant. The correlation between levels of care rationing and length of time practising as a registered nurse and length of time spent on current ward was the same as the critical care/emergency and medical specialities; however for the surgical specialty neither correlation was significant.

For the Surgical speciality a multiple regression analysis was not undertaken as none of the predictor variables were significant.

\section{Discussion}

Increased demands on the nursing workforce have restricted the amount of time a nurse can dedicate to each individual patient within their care. As a result, nurses ration the nursing care that they provide while ensuring patient safety as well as effectively meeting primary treatment goals. The aim of this current study was to determine the elements of care that are commonly rationed, and the reasons for nursing care rationing within the medical, surgical and critical care area specialties of an acute care hospital. Similarities and differences between the specialities were identified.

Monitoring of vital signs was performed more than $87 \%$ of the time and was the least rationed care in all three specialities. This result is not surprising as monitoring vital signs is a core function of the registered nurse and is an essential component of patient assessment and a determinant to the patient's health status ${ }^{31}$. An alteration in a patient's vital signs can provide objective evidence of the body's response to physical and psychological stress or changes in physiological function.

Hand hygiene is a simple and important method for preventing infections within the hospital environment.

Within the medical and surgical wards in this study hand washing was an element of care that was performed on a frequent basis, being rationed less than ten percent of the time. In comparison hand washing was rationed thirty percent of the time within the critical care specialty. The results ascertained from the critical care specialty are congruent with those from other studies that have utilized the MISSCARE scale to measure care rationing, with hand washing reported as rationed from $30 \%$ of the time $e^{15,30}$ up to $40.8 \%^{32}$ and $48.4 \%^{30}$ of the time. This could possibly be related to the predominant staff allocation model of care within critical care wards 
at the study hospital which sees a ratio of one critical care patient to one nurse. Nurses caring for the patient may not feel the need to wash hands as frequently between interventions as they are dealing with the same patient. On the other hand, it could be postulated that nurses could be using hand sanitizers and might have not considered it as hand washing.

Administering prn medications within 15 minutes and assessing the effectiveness of medications was identified as care that was least rationed by critical care/emergency and surgical nurses. In contrast nurses from medical wards administered prn medications within 15 minutes but assessing the effectiveness of medications was rationed. This may be attributed to the variance in what could be considered, the "routine" mode for medication administration in the specialty groups. In surgery, for example prn medication is a regular method of medication administration and often used in pain management.

Providing emotional support for the patient and their family was one of the elements of care that was least rationed in medical wards. This could be because it is not unusual for medical patients to have chronic conditions requiring frequent interactions with family as part of the episode of care and planning for post discharge.

Maintaining skin integrity and ensuring that meals were set up for patients who fed themselves were elements of care least rationed in critical care/emergency units. In contrast, these elements of care were the most frequently rationed in medical and surgical wards. This may in part be due to the greater nurse to patient ratio in medical and surgical wards and the difficulty in attending to multiple patients requiring assistance with meal preparation. Nurses may therefore ration such care that could be undertaken by patient carers such as oral care or assisting with meal set-up and feeding. Maintenance of skin integrity may be rationed on the basis that patients are more frequently being placed on pressure relieving devices. Nurses may assume that regular routine management of skin integrity is no longer required in the presence of a pressure relieving device.

Attendance at interdisciplinary care conferences occurred more frequently in surgical and medical specialities. This may be due to the fact that surgical and medicine specialties at the study site hold specific daily 
interdisciplinary care conferences to enhance the planning of care. In the critical care/emergency specialty this is performed as a ward round at the bedside and may not have been interpreted as a defined case conference by this nursing group. Patient discharge planning and teaching occurred less frequently in surgical and medical specialities which could be due to the fact that the medical and surgical units were more likely to have care coordinators and clinical nurse educators within the units with specific portfolios for patient flow management and education.

It should be noted that the way in which registered nurses ration or decide on a hierarchy of nursing care may be influenced by factors such as patient acuity, staffing levels, patient volume, the availability of resources, rate of patient turnover and the level of team work. ${ }^{30}$ In addition other factors include utilization of multidisciplinary team members such as social workers to provide emotional support to family members or a discharge planner to organize care post discharge. In addition, it is likely that the completion of some tasks such as patient education is primarily the responsibility of more specialist nurses such as clinical nurse educators or clinical nurse consultants during business hours.

Variances in care rationing between specialities may be explained by the differing natures of patients within each specialty. Patients in surgical wards undergo either planned elective or emergency surgical procedures. Patients undergoing a planned procedure tend to be well prior to attending hospital due to pre-operative screening procedures, and as such tend to maintain independence in the performance of self-care activities during their admission. Furthermore, the attendance of patients undergoing planned procedures aids in the processes of bed management and staffing allocation. In contrast patients in medical wards are unwell; suffering from acute and chronic illness/diseases that can be further complicated by the presence of co-morbidities making their trajectory is less predictable than that of surgical patients. Due to the fact that they are unwell, medical patients may be more reliant on nursing staff for self-care activities. Emergency departments have a high turnover of patients with patients being admitted to a ward or discharged home typically within four hours of presentation, it would therefore be expected in the context of this study, that less care is required to be provided within the emergency department. Patients within the critical care setting are highly dependent on nursing staff for all aspects of their care. While patient safety is maintained with low nurse to patient ratios, the technology laden environment and high acuity of patients may lead to the rationing elements of basic care, as reflected in this study. 
The main strength of the study was the inclusion of only registered nurses and clinical nurse specialists who provide direct patient care in the clinical setting. The nursing workforce at the study hospital consists predominantly of registered nurses and the perceptions of nursing staff within designations that provide less frequent to no direct patient care (clinical nurse educator, nurse educator, clinical nurse consultant, nurse unit manager) may have skewed the data. Furthermore, the survey was completed anonymously and participants were asked to comment about their practice environment as whole, rather than individual practices, promoting honesty and open disclosure without the fear of blame or repercussion. However, there is potential for recall bias and possible over reporting of missed care with nurses reflecting on the previous shift and workload.

This study was conducted in a single hospital that has a high proportion of registered nurses; therefore the results may not be generalizable to other hospitals that employ a larger proportion of enrolled and assistant nurses. A larger national study is warranted to investigate the similarities and differences in care rationing among rural and metropolitan hospitals and hospitals with a lower proportion of registered nurses. It should also be acknowledged that the nursing care tasks itemized in this study are not exhaustive, with nurses frequently performing tasks that have not been identified in the questionnaire. The combination of data from critical care units and the emergency department has made interpretation of results difficult due to the differing natures of the units and should be avoided in future studies.

\section{Conclusions}

The overall results from the study are consistent with current literature indicating that nurses are frequently unable to deliver all necessary aspects of patient care. This study demonstrates that here are numerous similarities and differences in care rationing between critical care/emergency, surgical and medical nurses. To meet the different requirements of different specialities individualized strategies should be developed to reduce the incidence of nursing care rationing. 


\section{Table legend}

Table 1: Demographic characteristics of the participants

Table 2: Percentage of care that is missed

Table 3: Univariate analysis to identify predictors of missed care

Table 4: Regression model to identify predictors of missed care 


\section{References}

1. Klein R, Day P. Rationing Health Care: The dilemma of Choice. In: Marmor TR, Klein R, eds. Politics, Health, and Health Care: Selected Essays. New Haven and London: Yale University Press, 2012:380.

2. Rumbold B, Alakeson V, Smith PC. Rationing health care: is it time to set out more clearly what is funded by the NHS. London: Nuffield Trust 2012. Available at

http://www.nuffieldtrust.org.uk/sites/files/nuffield/publication/rationing_health_care_240212.pdf. Accessed September 2015.

3. Papastavrou E, Andreou P, Efstathiou G. Rationing of nursing care and nurse-patient outcomes: a systematic review of quantitative studies. The Int J Health P Man. 2014;29:3-25.

4. Papastavrou E, Andreou P, Tsangari H, et al. Linking patient satisfaction with nursing care: the case of care rationing - a correlational study. BMC Nurs. 2014;13:26.

5. Ausserhofer D, Zander B, Busse R, et al. Prevalence, patterns and predictors of nursing care left undone in European hospitals: results from the multicountry cross-sectional RN4CAST study. BMJ Qual Saf. 2014;23:126-35.

6. Schubert M, Clarke S, Glass T, et al. Identifying thresholds for relationships between impacts of rationing of nursing care and nurse- and patient-reported outcomes in Swiss hospitals: a correlational study. Int J Nurs Stud. 2009;46:884 - 93 .

7. Bittner NP, Gravlin G, Hansten R, et al. Unraveling care omissions. J Nurs Adm. 2011;41:510-2.

8. Tschannen D, Kalisch BJ, Lee KH. Missed nursing care: the impact on intention to leave and turnover. Can J Nurs Res. 2010;42:22-39.

9. Gravlin G, Bittner NP. Nurses' and nursing assistants' reports of missed care and delegation. J Nurs Adm. 2010;40:329-35.

10. Kalisch BJ, Landstrom GL, Hinshaw AS. Missed nursing care: a concept analysis. $J$ Adv Nurs. 2009;65:1509-17.

11. Bittner NP, Gravlin G. Critical thinking, delegation, and missed care in nursing practice. J Nurs Adm. 2009;39:142-6.

12. Kalisch BJ. Missed nursing care: a qualitative study. J Nurs Care Qual. 2006;21:306-13.

13. Jones TL, Hamilton P, Murry N. Review: Unfinished nursing care, missed care, and implicitly rationed care: State of the science review. Int J Nurs Stud. 2015;52:1121-37.

14. Berg G, Spaeth D, Sook C, et al. Trauma patient perceptions of nursing care: relationships between ratings of interpersonal care, technical care, and global satisfaction. J Trauma Nurs. 2012;19:104 - 10.

15. Kalisch B, Landstrom G, Williams R. Missed nursing care: errors of omission. Nurs Outlook. 2009;57:3 - 9.

16. Kalisch B, Tschannen D, Lee KH. Missed nursing care, staffing, and patient falls. J Nurs Care Qual. 2012;27:6 - 12 .

17. Australian Institute of Health and Welfare. Admitted patient care 2013 - 14: Australian hospital statistics. 2015. Available at http://www.aihw.gov.au/WorkArea/DownloadAsset.aspx?id=60129550480. Accessed September 2015.

18. Carville O. Overworked nurses ration patient care. 2014. Available at http://www.stuff.co.nz/thepress/news/10079520/Overworked-nurses-ration-patient-care. Accessed September 2015.

19. Kalisch BJ, Xie B, Dabney BW. Patient-reported missed nursing care correlated with adverse events. Am J Med Qual. 2014;29:415-22. 
20. Lucero R, Lake E, Aiken L. Nursing care quality and adverse events in US hospitals. J Clin Nurs. 2010;19:2185- 95 .

21. Suhonen R, Papastavrou E, Efstathiou G, et al. Patient satisfaction as an outcome of individualised nursing care. Scand J Caring Sc. 2012;26:372 - 80.

22. Brooks-Carthon J, Kutney-Lee A, Sloane D, et al. Quality of care and patient satisfaction in hospitals with high concentrations of black patients. J Nurs Scholarsh. 2011;43:301 - 10.

23. Carthon JMB, Lasater KB, Sloane DM, et al. The quality of hospital work environments and missed nursing care is linked to heart failure readmissions: a cross-sectional study of US hospitals. BMJ Qual Saf. 2015;24:25563.

24. Ball JE, Murrells T, Rafferty AM, et al. 'Care left undone' during nursing shifts: associations with workload and perceived quality of care. BMJ Qual Saf. 2013;0:1-10. doi:10.1136/bmjqs-2012-001767.

25. Papastavrou E, Andreou P, Vryonides S. The hidden ethical element of nursing care rationing. Nurs Ethics. 2014;21:583-93.

26. Papastavrou E. The ethical complexities of nursing care rationing. J Nurs Adm. 2010;40:329-35.

27. Flinkman M, Isopahkala-Bouret U, Salanterä S. Young Registered Nurses' Intention to Leave the Profession and Professional Turnover in Early Career: A Qualitative Case Study. Int Sch Res Notices. 2013;19:1-12. doi: $10.1155 / 2013 / 916061$

28. Eley D, Eley R, Rogers-Clark C. Reasons for entering and leaving nursing: an Australian regional study. Aust J Adv Nurs. 2010;28:6-13.

29. Polis S, Higgs M, Manning V, et al. Factors contributing to nursing team work in an acute care tertiary hospital. Collegian. 2015. http://dx.doi.org/10.1016/j.colegn.2015.09.002.

30. Kalisch BJ, Williams RA. Development and psychometric testing of a tool to measure missed nursing care. $J$ Nurs Adm. 2009;39:211-9.31.

31. Elliott M, Coventry A. Critical care: the eight vital signs of patient monitoring. Br J Nurs. 2012;21:621-5.

32. Kalisch BJ, Terzioglu F, Duygulu S. The MISSCARE Survey-Turkish: psychometric properties and findings. Nurs Econ. 2012;30:29-37. 


\section{Acknowledgements}

Julie Cosgrove

St George Hospital, Executive Unit, Kogarah, New South Wales, Australia 